\title{
Left Apical Aneurysm in a Patient with Severe Aortic Valve Stenosis
}

\author{
Thorsten Drews ${ }^{1}$ Miralem Pasic ${ }^{1} \quad$ Roland Hetzer ${ }^{1}$ \\ ${ }^{1}$ Deutsches Herzzentrum Berlin, Berlin, Germany \\ Address for correspondence Thorsten Drews, MD, MBA, PhD, \\ Department of Cardiothoracic and Vascular Surgery, Deutsches \\ Thorac Cardiovasc Surg Rep 2014;3:9-12. \\ Herzzentrum Berlin, Augustenburger Platz 1, Berlin 13353, Germany \\ (e-mail: dr.t.drews@gmx.de).
}

\begin{abstract}
Keywords

- aneurysm

- heart diseases

- hypertension

- valves

We report on a very rare case of left ventricular aneurysm in a 77-year-old patient with aortic valve stenosis and without coronary artery disease. The patient underwent conventional aortic valve replacement and left ventricular aneurysmectomy with an uneventful postoperative course. The cause of the left ventricular aneurysm was suspected to be a long history of aortic valve stenosis that led to severe intraventricular hypertension, subsequently asymmetric septum hypertrophy, and finally apical aneurysm.
\end{abstract}

\section{Introduction}

A 77-year-old woman (height: $157 \mathrm{~cm}$, weight: $58 \mathrm{~kg}$ ) with a body surface area of $1.57 \mathrm{~m}^{2}$ suffered from increasing adynamia, dyspnea during exertion, and repeated syncope. The patient had been healthy for several years; she had stopped nicotine abuse due to increasing chronic obstructive lung disease with chronic dry cough. A cerebral infarction 6 months before had led to intensive physical examinations, and severe aortic valve failure was found. Due to the patient's advanced age and chronic lung disease, the operative risk was assessed as high according to the logistic EuroSCORE and EuroSCORE II of 18.21 and $7.4 \%$, respectively. The patient was transferred to our institution for evaluation of transapical or transfemoral aortic valve implantation.

On admission, transthoracic and transesophageal echocardiography confirmed the diagnosis of severe aortic valve stenosis (aortic valve area, $0.79 \mathrm{~cm}^{2}$; maximal transvalvular gradient, $80 \mathrm{~mm} \mathrm{Hg}$; mean transvalvular gradient, $50 \mathrm{~mm}$ $\mathrm{Hg}$ ) and valve incompetence of grade I. The mitral and tricuspid valves also showed slight incompetence of grade I. Echocardiographic examination revealed normal right and left ventricular contractility. Left ventricular end-systolic and end-diastolic diameters were 30 and $50 \mathrm{~mm}$, respectively. The left ventricle was hypertrophic with myocardial systolic and diastolic thicknesses of the interventricular septum of 16 and $13 \mathrm{~mm}$, respectively. The maximum subvalvular gradient due to septum hypertrophy was $10 \mathrm{~mm} \mathrm{Hg}$. The apex of the left ventricle was dyskinetic with a 30-mm apical aneurysm (-Fig. 1a). The wall thickness of the aneurysm was approximately $3 \mathrm{~mm}$. Coronary artery disease was excluded by coronary angiography (-Figs. 2-4). Catheter examination with left ventriculography confirmed the diagnosis of aortic valve stenosis (peak-to-peak transvalvular gradient, maximal $64 \mathrm{~mm} \mathrm{Hg}$; mean, $40 \mathrm{~mm} \mathrm{Hg}$ ) and left ventricular apical aneurysm (-Fig. 5). In computed tomography of the chest, the aneurysm was measured and its cohesion to neighboring structures was visualized (-Figs. 6a, b).

The situation was first evaluated by our staff and then discussed with the patient. Transapical valve implantation was abandoned and a conventional operation with aortic valve replacement and myocardial aneurysm resection was decided upon. The patient's written informed consent was obtained (as standard, obligatory procedure in Germany).

We used our standard surgical strategy with median sternotomy and aortic and right atrial cannulation for the heartlung machine with warm antegrade potassium blood cardioplegia. ${ }^{1}$ Under cardiac arrest, the apical aneurysm was clearly palpated. The thin apical wall of $3 \mathrm{~mm}$ was opened and resection with reconstruction of the geometry of the left ventricle was performed. The left ventricular cavity was explored through the aneurysm. There was only one place with asymmetric septal hypertrophy, at the aneurysmal base. Afterward the calcified, native aortic valve was resected and replaced by a biological xenograft prosthesis (Hancock, received

December 29, 2013

accepted after revision

February 27, 2014

published online

May 6, 2014
DOI http://dx.doi.org/

10.1055/s-0034-1374806. ISSN 2194-7635. (c) 2014 Georg Thieme Verlag KG Stuttgart · New York
License terms

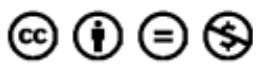


a
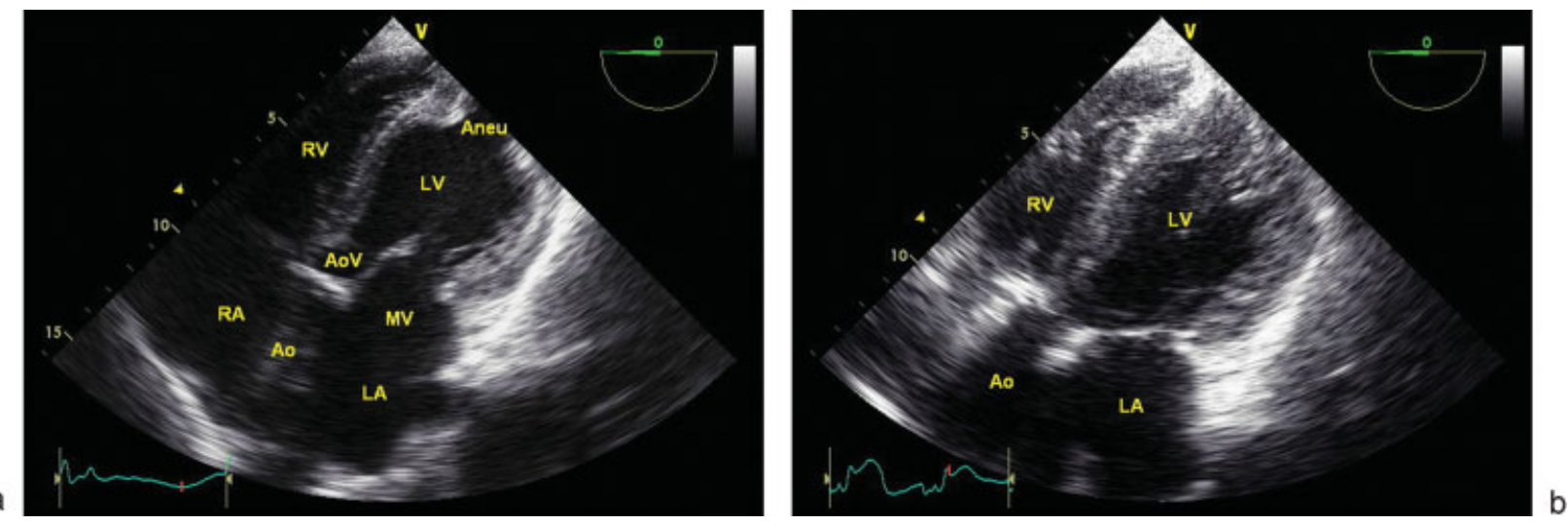

Fig. 1 (a) Echocardiography of the heart in end diastole (preoperative). (b) Echocardiography of the heart in end diastole (postoperative).

$23 \mathrm{~mm}$; Medtronic, Minneapolis, Minnesota, United States). The postoperative course was uneventful. The patient was extubated on the 1st postoperative day, transferred to the normal ward, and discharged from hospital on the 9th postoperative day. Postoperative echocardiographic examination showed excellent prosthetic xenograft valve function and normal left ventricular function without apical aneurysm (-Fig. 1b). The histological findings of the apical tissue revealed transmural fibrosis, including local calcification and a significant loss of cardiomyocytes ( - Fig. 7). Coronary vessels in the subepicardial fat and the connective tissue showed fibrosis of the vessel wall and obliterated lumen of some of the vessels. Due to the reduction in the number of myocardial cells, there were no findings of disorganization of cells as indices for hypertrophic obstructive cardiomyopathy (HOCM).

\section{Commentary}

Left ventricular aneurysms are usually observed in patients with ischemic myocardium, following infarction with pres-

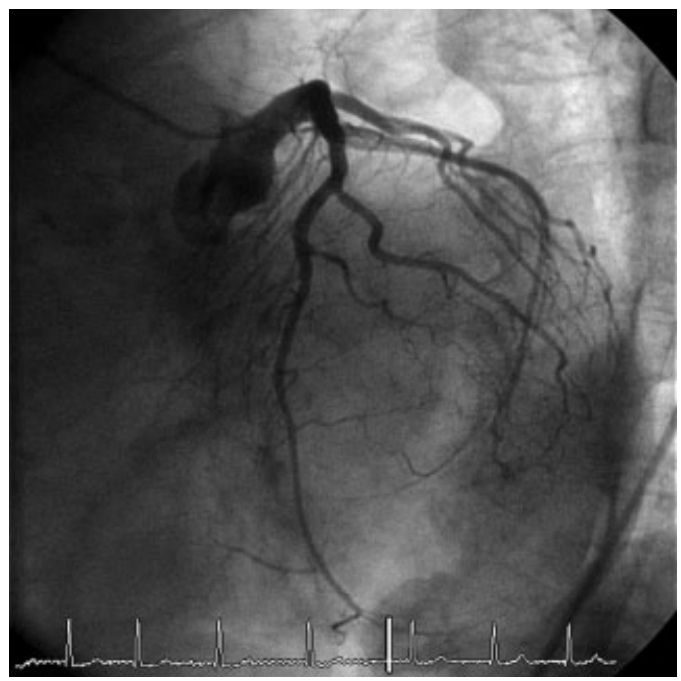

Fig. 2 Coronary angiography with exclusion of calcification of the left main coronary artery. sure impact during or after cicatrization. Rarely, they can be found in patients with HOCM. ${ }^{2-4}$

We report on a patient with aortic valve stenosis and left ventricular apical aneurysm of unclear cause. Histological examination excluded HOCM and other possible causes for aneurysm formation were also excluded, such as coronary artery disease, myocardial infarction, severe systemic hypertension, Chagas disease, or other inflammatory and traumatic diseases. ${ }^{5}$ We believe that the possible cause of the aneurysm in our patient was that a long history of aortic valve stenosis had led to severe intraventricular hypertension, subsequently to asymmetric septal hypertrophy, and finally to apical aneurysm. Asymmetric septal hypertrophy can be observed in elderly patients with severe aortic stenosis. It is mostly located below the aortic valve, in the left ventricular outflow tract, but not deep in the left ventricle, as in our patient.

Apical aneurysms are mostly observed in patients with HOCM. Maron et $\mathrm{al}^{3}$ analyzed the data of 1,299 patients with HOCM. In 28 patients (2\%), an apical aneurysm was observed.

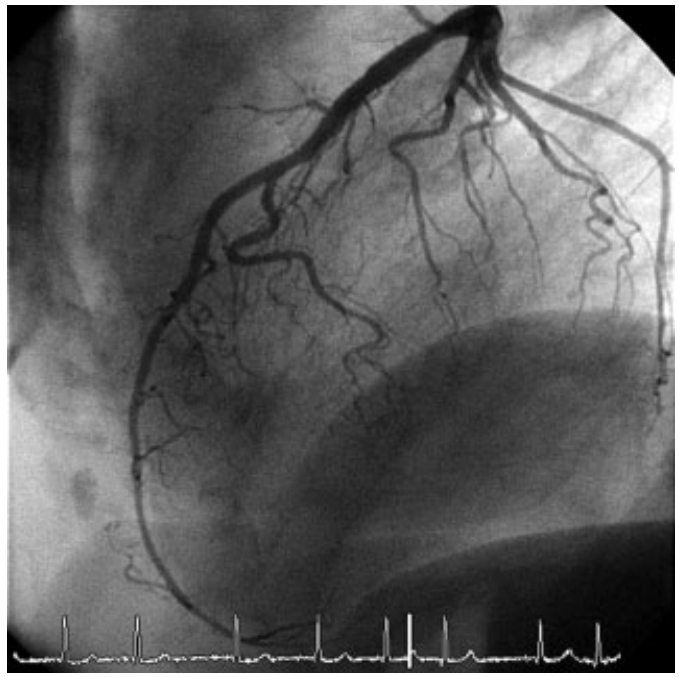

Fig. 3 Coronary angiography with exclusion of calcification of the left anterior descending. 


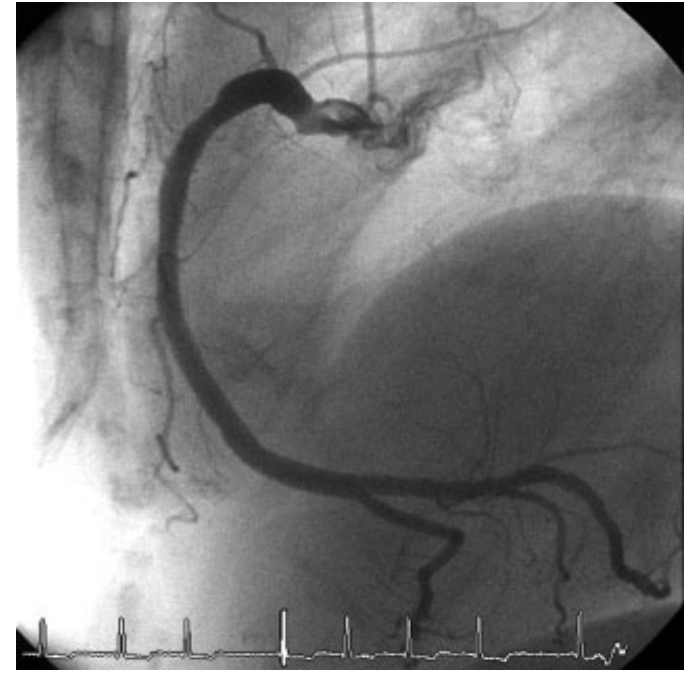

Fig. 4 Coronary angiography with exclusion of calcification of the right coronary artery.

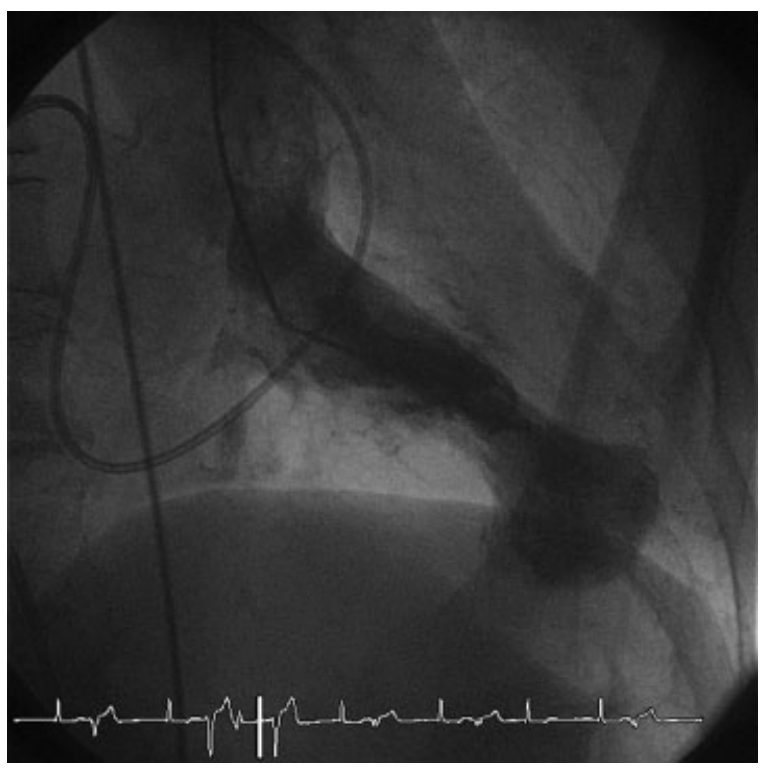

Fig. 5 Angiography of the left ventricle in end systole (preoperative).

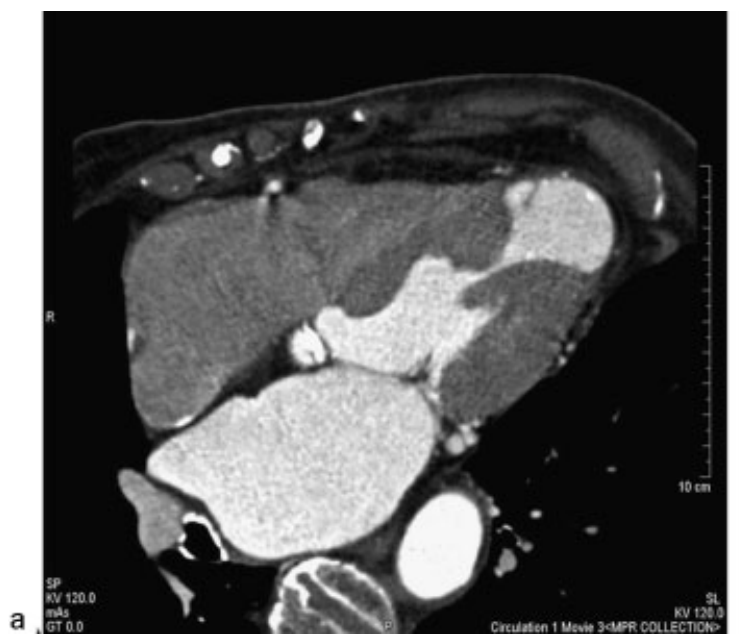

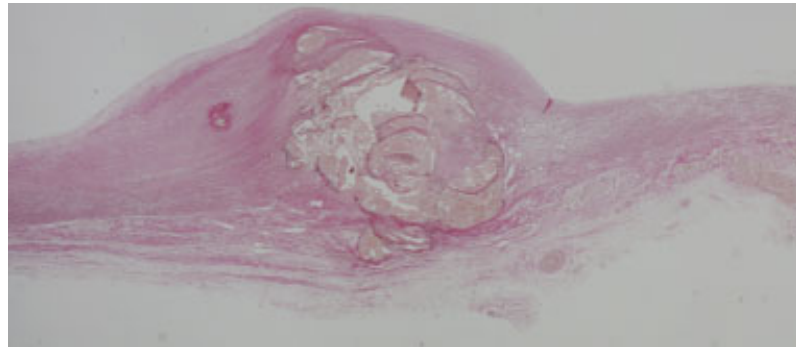

Fig. 7 Histological examination of the apical tissue (Elastic van Gieson, 50x).

The prevalence did not correlate with age, sex, or sarcomeric protein mutations. Twelve of them (43\%) presented complications (sudden death, stroke, etc.). Our patient also had a history of stroke. Nevertheless, she had no HOCM. Soufen et al described an apical aneurysm with septum hypertrophy in a patient with severe systemic hypertension without history of HOCM. ${ }^{2}$ Their patient, a 59-year-old woman, had in her history isolated hypertension and cerebral embolism. She had an apical aneurysm with a thrombus leading to long-term oral anticoagulation with warfarin. ${ }^{2}$ In Brazil, the presence of an apical aneurysm is always suggestive of Chagas disease. ${ }^{6}$ Our patient was from northern Europe and had no history of visits to Central or South America. In addition, she had no symptoms of intestinal hypomotility, as seen in patients with Chagas disease, so that this correlation could be effectively excluded.

We considered the patient for transapical or transfemoral aortic valve implantation. As a left ventricular apical aneurysm was diagnosed, we decided to use a standard technique with median sternotomy and cardiopulmonary bypass. Transapical valve implantation with access to the apex of the heart by mini anterior left thoracotomy in the fifth intercostal space and simultaneous aneurysm resection seemed possible. Theoretically, it would have been possible to perform transapical aortic valve implantation through the apical aneurysm with concomitant exclusion of the apical aneurysm (aneurysmorrhaphy) without use of cardiopulmonary bypass. In contrast, transfemoral valve implantation might have carried a possible risk of perforation of the apical aneurysm by retrograde introduction of

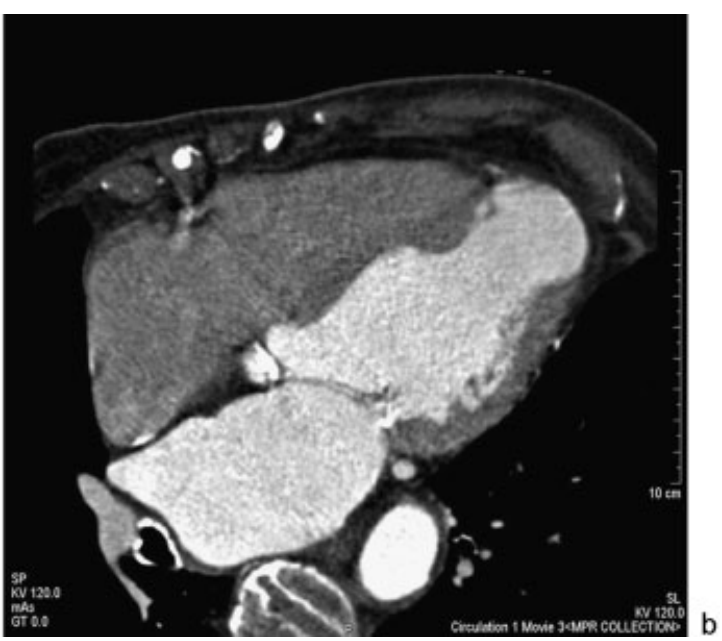

Fig. 6 (a) Computed tomography of the heart in end diastole (preoperative). (b) Computed tomography of the heart in end systole (preoperative). 
12 Left Apical Aneurysm in a Patient with Severe Aortic Valve Stenosis Drews et al.

the stiff guide wire into the left ventricle. Moreover, aneurysm resection during the same operative session would have required additional thoracic opening or that the aneurysm be left in situ. Following the European Society of Cardiology guidelines, left ventricular aneurysm may result in systolic and diastolic dysfunction and mural thrombus formation. ${ }^{7}$ Therefore, the indication for aneurysmectomy was given not only due to the risk of spontaneous perforation with tamponade but also due to the risk of resistant arrhythmias and of potential peripheral embolism. ${ }^{5}$

\section{Acknowledgment}

The authors thank Anne Gale, ELS (Editor in the Life Sciences) for editorial assistance.

\section{Disclosures}

T. Drews and M. Pasic served as proctors to Edwards Lifesciences from July 2009 to June 2012. There are no other disclosures.

\section{References}

1 Calafiore AM, Teodori G, Di Giammarco G, et al. Intermittent antegrade cardioplegia: warm blood vs cold crystalloid. A clinical study. J Cardiovasc Surg (Torino) 1994;35(6, Suppl 1):179-184

2 Soufen HN, Frimm Cd, Benvenuti LA, Mady C. Apical aneurysm and left ventricular hypertrophy. Arq Bras Cardiol 2000;75(2):145-150

3 Maron MS, Finley JJ, Bos JM, et al. Prevalence, clinical significance, and natural history of left ventricular apical aneurysms in hypertrophic cardiomyopathy. Circulation 2008;118(15):1541-1549

4 Teraoka K, Hirano M, Ogawa T, et al. Hypertrophic cardiomyopathy with mid-ventricular obstruction complicated by apical aneurysm appearing as delayed contrast hyperenhancement on magnetic resonance imaging: two case reports [in Japanese]. J Cardiol 2003; 42(2):87-94

5 Rimailho A, Cabrol C, Soyer R, et al. Idiopathic aneurysm of the left ventricle. Apropos of 4 cases operated on with success [in French]. Arch Mal Coeur Vaiss 1981;74(4):443-451

6 Oliveira JS, Mello De Oliveira JA, Frederigue U Jr, Lima Filho EC. Apical aneurysm of Chagas's heart disease. Br Heart J 1981;46(4):432-437

7 Steg PG, James SK, Atar D, et al; Task Force on the management of ST-segment elevation acute myocardial infarction of the European Society of Cardiology (ESC). ESC Guidelines for the management of acute myocardial infarction in patients presenting with STsegment elevation. Eur Heart J 2012;33(20):2569-2619 